\title{
Effects of Sampling Rate on Collision Probability in Hybrid MAC Protocols in WSN
}

\author{
Qian Dong, Waltenegus Dargie and Alexander Schill \\ Chair of Computer Networks, Faculty of Computer Science, Technical University of Dresden, Germany, 01062 \\ Email: qian.dong, waltenegus.dargie, alexander.schill@tu-dresden.de
}

\begin{abstract}
In wireless sensor networks, energy is a scarce resource. Collision as one of the major sources of the energy waste for medium access control (MAC) protocols has been extensively investigated. However, it is mostly analyzed under the "saturated situation" in which the sampling rate is assumed to be equal to or larger than the transmission rate, which does not reflect the reality in many applications. This paper relaxes this assumption to consider the "unsaturated situation" in which nodes do not always contend to access the media. It focuses on hybrid MAC protocols which enable nodes to define sleeping schedules. We take SMAC as a model protocol to analyze how the sampling rate affects the probability of collision. But the approach can easily be applied to other hybrid protocols such as BMAC and XMAC.
\end{abstract}

Keywords: Collision, MAC protocols, Wireless Sensor Networks, Control Packet Overhead, CSMA/CA

\section{INTRODUCTION}

Wireless sensor networks are one of the popular technologies of the future due to a wide range of application areas [1] [2] [3] [4]. Since it is difficult to change or recharge batteries of sensor nodes, energy efficiency is the biggest issue to be considered. Collision, as the source of the retransmission, highly influences the total energy consumption of a MAC protocol.

In recent years, the collision probability in wireless MAC protocols (particularly, in IEEE 802.11) has been extensively studied due to its impact on throughput and delay [5] [6] [7] [8]. However, most of these studies assume a "saturated situation" where the sensor nodes always have packets to send. This assumption, however, does not reflect the reality in many applications. What usually happens is in fact the unsaturated situation in which the sampling rate is much less than the transmission rate. As a result, nodes can be idle for a long period before the newly sampled data arrive. Hence, it is not necessary to keep nodes listening all the time.

Hybrid protocols define the periodic sleeping schedule to minimize the idle time of wireless sensor nodes. However, the collision probability in these protocols is not well studied. Intuitively, the probability of collision may decrease due to the lower sampling rate in hybrid protocols. The diminished collision probability, however, poses the question whether control packets are still necessary to be adopted in hybrid MAC protocols in terms of energy expenditure. This paper investigates the overall collision probability (both in the case of saturated and unsaturated situations) from the perspective of the sampling rate in hybrid MAC protocols. The contention model is analyzed based on the SMAC protocol [9], but it can be easily generalized. The analytical model assumes a finite number of terminals, ideal channel conditions, independent collision probability of packets and infrequent communication among sensor nodes.

The rest of the paper is organized as follows: in section 2 , related work is introduced. In section 3 , the listen phase of the SMAC protocol is analyzed. In section 4, the overall collision probability is evaluated by averaging the collision probability in both the saturated and unsaturated situations. In section 5 , the characteristic of the overall collision probability is visualized and the observations are discussed. Finally in section 6 , concluding remarks are given.

\section{RELATED WORK}

Collision, as one of the major energy consumption sources, has been the focus of an extensive research, especially in IEEE 802.11 protocol. Bianchi uses the Markov Chain model to calculate the collision probability in the saturated situation in order to compute the throughput [5]. The impact of channel capture following a busy period on collision probabilities in the saturated network is studied in [6]. While a box-ball model [7] is developed to resolve the probability of packet collisions so that the power consumption in the saturated WLAN environment can be evaluated. Obviously, all these approaches concentrate only on the saturated situation of the 802.11 DCF specification with the assumption that the sampling rate is not less than the transmission rate, which, however, does not reflect the reality in many wireless sensor applications.

The model of Malone et al. [10] improves the Bianchi model by considering more realistic networks in which the data traffic is considered to be non-saturated and the packet arrival rate is non-uniform. The model enables to estimate the peak throughput. The analytic model of Kumar et al. [11] is similar to that of Malone et al., but it considers additional aspects such as channel access rate and models the backoff by a discrete time Markov chain (DTMC). Likewise, Bruno et al. [8] analyze the efficiency and energy consumption of wireless networks by employing a p-persistent carrier sense multiple access scheme. Their model establishes a relationship between node density, collision rate and packet loss such that the optimal $p$ - a parameter that determines the average size of the contention window - can be computed. The analysis result shows that besides maximizing throughput, $p$ also minimizes 
the network's energy consumption. All these approaches, however, only focus on wireless local area networks in which the energy consumption of nodes is not the main concern and therefore the nodes are active all the time.

\section{Analysis of the Listen Phase}

\section{A. Saturated and Unsaturated}

A hybrid protocol in wireless sensor networks adopts a medium access strategy based on contention and a periodic sleep schedule. During contention, after the final ACK frame is successfully transmitted, the channel becomes free and a new round of competition starts. At this time, all the neighbors of the transmitter and the receiver will finish waiting and begin to contend for the medium. If these neighbors have some available sampled packets to transmit, they will contend for the medium immediately by first monitoring the channel for a period of DIFS. Therefore, in the saturated period, the DIFS interval immediately follows the previous final ACK frame. In other words, the time interval $T$ (as shown in Fig. 1(a)) between the end of the previous ACK frame and the beginning of the new DIFS sensing period equals zero. In the unsaturated period, however, a node may not have data to transmit at the moment. Therefore, $T$ (as shown in Fig. 1(b)) is larger than zero. The fundamental reason for the difference between the saturated and unsaturated situations is the relationship between the sampling and the transmission rates. In the former case, the sampling rate is close to or larger than the transmission rate. But in the latter case, the sampling rate is much smaller than the transmission rate.

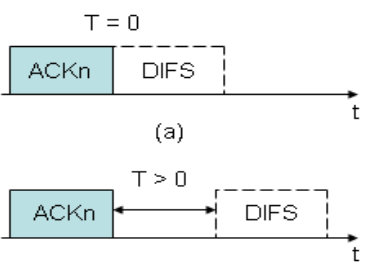

(b)

Fig. 1. Saturated and unsaturated situations with regard to $\mathrm{T}$.

\section{B. Listen Phase Division}

Another aspect of hybrid MAC protocols is the periodic sleep which enables nodes to minimize the energy consumption of idle listening. To reduce control overhead, neighbors synchronize their sleep schedules. This is accomplished by transmitting a SYNC packet periodically at the beginning of the listen period after the carrier sense. If the channel is detected free during the DIFS period, a randomly chosen backoff begins to decrease and transmission starts when it reaches zero.

Since the sensing task does not stop during the sleep phase, some packets will be accumulated in each node's queue before the next listen phase arrives. Hence, in the first part of the saturated period ( $T_{0}$ in Fig. 2), nodes will transmit all these packets at the nodes' transmission rate. As the sensing task is also taking place in the saturated period (summation of $T_{0}$ and $T_{1}$ ), several newly sampled packets will be stored in each node's queue, and nodes should also keep transmitting these packets until the queues become empty. At this time point (at the end of $T_{1}$ in Fig. 2), all the data packets sampled during both $T_{0}$ and $T_{1}$ are transmitted and no packet will be accumulated in the queues anymore. So once a new sample arrives, the node will send it out immediately. In other words, the unsaturated period starts.

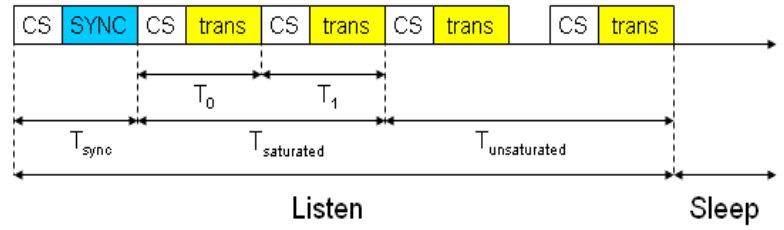

Fig. 2. The characteristics of the listen phase.

The saturated period must appear in each listen phase since the duty cycle is always smaller than one even though the sampling rate is smaller than the transmission rate. In addition, the unsaturated period must exist as well although we want to reduce the idle listening as much as possible. The reason is that some extra time must be spared for carrying out the retransmission caused by collision and errors during the data transmission. Since the number of packets which will be retransmitted as well as the number of retransmission for a packet is random, the unsaturated period should be set long enough to deal with retransmission problems.

\section{Overall Collision Probability Model}

Unlike the way that the collision probability is studied either in the saturated or unsaturated situation in the 802.11 protocol, the collision probability for hybrid MAC protocols should be averaged into an overall one. Such average method is operated in terms of the total number of data packets transmitted during the saturated and unsaturated periods, since the number of data packets equals the number of RTS packets which are actually the objects of collision.

\section{A. Overall Collision Probability}

During the sleep period, all the sensor nodes will turn off their radios, so no data packet is transmitted. Hence, collisions will not occur. Subsequently, our focus is only on the listen phase. The symbols, $p_{s}$, and, $p_{u}$, are adopted to denote the collision probability in the saturated and unsaturated periods, respectively, and the overall collision probability, $p$, can be described as the arithmetic average of $p_{s}$ and $p_{u}$. Since the average scheme should be handled in terms of the number of data packets, the ratio of the number of data packets transmitted during the saturated period, $n_{s}$, to the total number of data packets transmitted during the whole listen phase should be used as the weight for the collision probability in the saturated period. In the same manner, the ratio of the number of data packets transmitted during the unsaturated period, $n_{u}$, to the total number of data packets transmitted during the 
whole listen phase should be chosen as the weight for the collision probability in the unsaturated period. Then the overall collision probability can be expressed as:

$$
p=p_{s} \frac{n_{s}}{n_{s}+n_{u}}+p_{u} \frac{n_{u}}{n_{s}+n_{u}}
$$

Since the sensing task never stops, the newly sampled data packets keep on arriving at the queue of each node during the whole frame (includes a synchronized, a saturated, an unsaturated and a sleep phase). Since no data transmission occurs during the sleep period, $T_{\text {sleep }}$, those accumulated in-queue packets can only be transmitted during the next synchronized, $T_{\text {sync }}$, and the first part of the saturated phases, $T_{0}$. Likewise, the data packets newly sampled during $T_{\text {sync }}$, $T_{0}$ and $T_{1}$ will be transmitted in $T_{1}$. Until then, the saturated phase, $T_{s a}$, completes. Consequently, the total number of data packets transmitted during the synchronized and saturated periods is equal to the number of data packets accumulated during the previous sleep, the current synchronized and the saturated periods. This can be evaluated by considering the sampling rate, $R_{s}$, as well as the data packet size, $N_{\text {data }}$.

$$
n_{s}=\frac{R_{s} T_{\text {sleep }}+R_{s}\left(T_{s a}+T_{\text {sync }}\right)}{N_{\text {data }}}
$$

However, synchronization is only maintained periodically in hybrid MAC protocols. Moreover, unlike the saturated period during which many data packets are transmitted, there is only one small SYNC frame sent in the synchronization interval. This indicates that the synchronization period is much shorter than the saturated period. Hence, the number of data packets sampled during the synchronization period is too small to be counted and thus is ignored. This also means that our model is valid to other types of hybrid MACs (such as BMAC [13] and XMAC [14]) which do not require schedule synchronization.

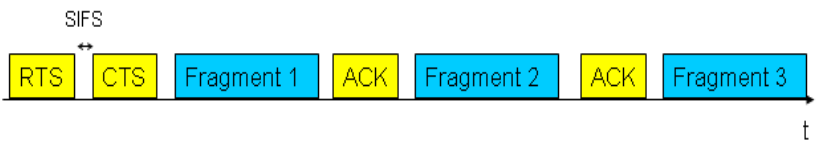

Fig. 3. Message passing mechanism.

Furthermore, the message passing mechanism is adopted in hybrid MAC protocols in order to reduce the retransmission overhead. The mechanism divides a long message into several small and independent fragments which will be transmitted in burst, as displayed in Fig. 3. Therefore, the size of a sampled data packet, $N_{\text {data }}$, equals the summation of the size of all its separated fragments, $N_{f}$. In this way, Equation (2) can be reformulated as:

$$
n_{s}=\frac{R_{s} T_{\text {sleep }}+R_{s} T_{s a}}{n N_{f}}
$$

As stated before, the end of $T_{1}$ indicates the beginning of the unsaturated period, during which the transmitted packets are obtained from data that are sampled during this period itself.
So the total number of the data packets transmitted during the unsaturated period, $T_{\text {unsa }}$, can be evaluated as:

$$
n_{u}=\frac{R_{s} T_{u n s a}}{n N_{f}}
$$

As shown in Fig. 2, the length of the unsaturated period equals the frame length excluding the length of the sleep and the saturated phases. Then equation (4) can be transformed to:

$$
n_{u}=\frac{R_{s}\left(T_{\text {frame }}-T_{\text {sleep }}-T_{\text {sa }}\right)}{n N_{f}}
$$

By inserting equations (3) and (5) into equation (1) and simplifying the result, we can observe that the overall collision probability is related to several time durations besides the collision probability in the saturated and unsaturated periods.

$$
p=\frac{p_{s}\left(T_{\text {sleep }}+T_{\text {sa }}\right)+p_{u}\left(T_{\text {frame }}-T_{\text {sleep }}-T_{\text {sa }}\right)}{T_{\text {frame }}}
$$

Here, the sleep phase, $T_{\text {sleep }}$, can be expressed by the frame time, $T_{\text {frame }}$, and the duty cycle, D:

$$
T_{\text {sleep }}=T_{\text {frame }}(1-D)
$$

Then equation (6) can be written as:

$$
p=\frac{p_{s}\left(T_{\text {frame }}(1-D)+T_{\text {sa }}\right)+p_{u}\left(T_{\text {frame }} D-T_{\text {sa }}\right)}{T_{\text {frame }}}
$$

Equation (8) expresses that the overall collision probability is dependent on several parameters. Among them, both $p_{s}$ and $p_{u}$ have already been successfully evaluated in [12]. $T_{\text {frame }}$ and $D$ are known already. The only unknown variable is $T_{s a}$ whose value cannot be determined directly. And the overall collision probability can be evaluated only if $T_{s a}$ can be resolved.

\section{B. Resolution of the Saturated Period}

As stated before, the data packets transmitted during the current $T_{0}$ come from the data packets sampled during the previous sleep phase. Thus the number of data packets, $N_{T_{0}}$, transmitted during $T_{0}$ can be described as:

$$
N_{T_{0}}=\frac{R_{s} T_{\text {sleep }}}{n N_{f}}
$$

During the time interval, $T_{0}$, the packets are transmitted in the saturated condition. This, however, does not mean that the time interval between every two transmission rounds is zero. Instead, there is a DIFS sensing period, a randomly chosen backoff time, several SIFS frames and control packets in the transmission, as shown in Fig. 4. Therefore, when evaluating the total time used for transmitting the data packets which are sampled during the previous sleep period, we should exclude the time spent on all these frames. The total time used for the backoff during $T_{0}$ can be estimated by considering a single backoff duration, $T_{a v g} \sigma$, and the total number of data packets 
transmitted during $T_{0}, N_{T_{0}}$, as the function $T_{T_{0}}^{\text {backoff }}$ expresses where $T_{\text {avg }}$ is the average backoff and $\sigma$ is a slot time.

$$
T_{T_{0}}^{\text {backoff }}=T_{\text {avg }} \sigma \frac{R_{s} T_{\text {sleep }}}{n N_{f}}
$$

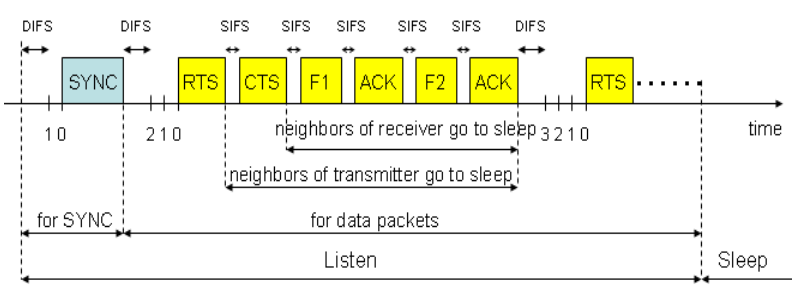

Fig. 4. Transmission characteristics of the saturated period.

Similarly, the total time, $T_{T_{0}}^{D I F S}$, spent on the DIFS sensing period during $T_{0}$, should be evaluated as:

$$
T_{T_{0}}^{D I F S}=T_{D I F S} \frac{R_{s} T_{\text {sleep }}}{n N_{f}}
$$

Usually, the error probability depends on the reliability of the wireless sensor network and is very small, so the time used for the retransmission of data fragments and control packets are not worth to be taken into consideration. Therefore, merely $(2 n+1)$ SIFS frames appear in a round of transmission, as one SIFS frame stands between every two neighbor packets. So the total time, $T_{T_{0}}^{S I F S}$, used for the SIFS frames during $T_{0}$ can be described as:

$$
T_{T_{0}}^{S I F S}=(2 n+1) T_{S I F S} \frac{R_{s} T_{\text {sleep }}}{n N_{f}}
$$

And the total time spent on the transmission of the control packets RTS, CTS and ACK during $T_{0}$ is expressed as function $T_{T_{0}}^{\text {control packet }}$ where $R_{t}$ denotes the transmission rate.

$$
T_{T_{0}}^{\text {control packet }}=\left(\frac{N_{r t s}}{R_{t}}+\frac{N_{c t s}}{R_{t}}+n \frac{N_{a c k}}{R_{t}}\right) \frac{R_{s} T_{\text {sleep }}}{n N_{f}}
$$

By combining equations (10) (11) (12) and (13), the total time, $T_{T_{0}}$, which is not directly used for the data packet transmission during $T_{0}$ can be calculated where $T_{\text {trans }}=$ $T_{a v g} \sigma+T_{D I F S}+(2 n+1) T_{S I F S}+\frac{N_{r t s}+N_{c t s}+n N_{a c k}}{R_{t}}$.

$$
T_{T_{0}}=T_{\text {trans }} \frac{R_{s} T_{\text {sleep }}}{n N_{f}}
$$

By subtracting $T_{T_{0}}$ from $T_{0}$, the time which is directly spent in transmitting the data packets that are sampled during the previous sleep phase can be estimated by function $T_{T_{0}}^{\text {data }}$.

$$
T_{T_{0}}^{\text {data }}=T_{0}-T_{\text {trans }} \frac{R_{s} T_{\text {sleep }}}{n N_{f}}
$$

Obviously, all the data transmitted during this remaining time, $T_{T_{0}}^{\text {data }}$, at the radio's transmission rate are sampled in the previous sleep phase. Therefore, we can set up an equation by making use of this relationship.

$$
\frac{R_{s} T_{\text {sleep }}}{R_{t}}=T_{0}-T_{\text {trans }} \frac{R_{s} T_{\text {sleep }}}{n N_{f}}
$$

The data packets that are transmitted in the second part of the saturated period, $T_{1}$, are sampled during the whole saturated period. Similar to the time interval $T_{0}$, part of the time within $T_{1}$ is spent on the carrier sense, the SIFS frames and the transmission of control packets. Such time duration, $T_{T_{1}}$, can be expressed as:

$$
T_{T_{1}}=T_{\text {trans }} \frac{R_{s} T_{s a}}{n N_{f}}
$$

Then by subtracting $T_{T_{1}}$ from $T_{1}$ (note that $T_{s a}$ is the summation of $T_{0}$ and $T_{1}$ ), the useful time duration which is directly spent on data transmission during $T_{1}$ can be estimated as:

$$
T_{T_{1}}^{\text {data }}=T_{s a}-T_{0}-T_{\text {trans }} \frac{R_{s} T_{s a}}{n N_{f}}
$$

Since the data sampled in the saturated period will be transmitted during $T_{T_{1}}^{\text {data }}$, we can set up another equation which expresses the relationship among the number of data packets sampled in the saturated period, the transmission rate, and this remaining time of $T_{1}$.

$$
\frac{R_{s} T_{s a}}{R_{t}}=T_{s a}-T_{0}-T_{\text {trans }} \frac{R_{s} T_{s a}}{n N_{f}}
$$

By combining equations (16) and (19) and simplifying the expression, the value of the saturated period can be successfully evaluated:

$$
T_{s a}=\frac{T_{\text {trans }} \frac{R_{s} T_{\text {sleep }}}{n N_{f}}+\frac{R_{s} T_{\text {sleep }}}{R_{t}}}{1-\frac{R_{s}}{R_{t}}-T_{\text {trans }} \frac{R_{s}}{n N_{f}}}
$$

\section{Resolution of the Collision Probability}

By inserting equation (20) into equation (8), it is possible to drive an expression for the overall collision probability. Equation (21) actually gives the relationship between the overall collision probability and the sampling rate in hybrid MAC protocols in wireless sensor networks.

$$
\begin{aligned}
p & =p_{s}\left((1-D)+\frac{T_{\text {trans }} \frac{R_{s}(1-D)}{n N_{f}}+\frac{R_{s}(1-D)}{R_{t}}}{1-\frac{R_{s}}{R_{t}}-T_{\text {trans }} \frac{R_{s}}{n N_{f}}}\right) \\
& +p_{u}\left(D-\frac{T_{\text {trans }} \frac{R_{s}(1-D)}{n N_{f}}+\frac{R_{s}(1-D)}{R_{t}}}{1-\frac{R_{s}}{R_{t}}-T_{\text {trans }} \frac{R_{s}}{n N_{f}}}\right)
\end{aligned}
$$

\section{Simulation And Discussion}

\section{A. Simulation Setup and Parameters}

Matlab version 7.0.1 was employed to verify and visualize the correlation between the overall collision probability and the node's sampling rate in both saturated and unsaturated conditions of hybrid MAC protocols. The analytic model was applied to a network in which nodes are randomly distributed 
in an area of $160 \times 200$ meters. One of these nodes is the information sink and it has endless power supply. We assume that the transmission rate is $2 \mathrm{Mbps}$ and the network operates under stationary and ideal channel conditions.

During the simulation, the transmission rate was expressed in terms of byte per microsecond in order to comply with the way DCF in IEEE 802.11 specification (the time unit of SIFS, DIFS and the slot time as well as the length unit of control packets and normal data packets) is expressed. Due to the limitation of the time interval, $T$, in hybrid MAC protocols, even the maximum sampling rate is much smaller than the transmission rate. The number of fragmentations for a single message is selected to be 4 . This makes the average size of a data fragment equal 34 bytes.

In this paper, the aim is to examine the influence of varying the sampling rate on the overall collision probability. Subsequently, how the other parameters affect the overall collision probability falls out of the scope. By doing so, all the other parameters should be set as constants throughout the simulation. The constant for the duty cycle, however, should be large enough to ensure that hybrid MAC protocols work properly even if the maximum allowed sampling rate is reached. All the other parameters are presented in Table I.

TABLE I

SimULATION PARAMETER List

\begin{tabular}{l|l}
\hline Basic Parameter & Default Value \\
\hline Control message RTS/CTS/ACK & 10 bytes \\
Data message & 136 bytes \\
Fragmentation & 4 \\
Transmission rate & $2 \mathrm{Mbps}$ \\
Maximum backoff stage & 5 \\
Maximum transmission attempt & 8 \\
Minimum contention window & 31 \\
Sensing field & $32000 \mathrm{~m}^{2}(160 \mathrm{~m} \times 200 \mathrm{~m})$ \\
Total sensor node & 50 \\
Nominal transmission range & $40 \mathrm{~m}$ \\
SIFS & $10 \mu \mathrm{s}$ \\
DIFS & $50 \mu \mathrm{s}$ \\
Slot time & $20 \mu \mathrm{s}$ \\
Duty cycle & $80 \%$ \\
\hline
\end{tabular}

\section{B. Simulation Results and Discussion}

As analyzed in [12], the collision probability in the saturated period is simulated as a straight line parallel to the axis of the sampling rate. Due to the limitation of the time interval, $T$, the maximum achievable sampling rate is $1.18 \mathrm{Mbps}$. On the other hand, the collision probability in the unsaturated period behaves differently when the sampling rate belongs to different conditions with respect to $T$. Since the overall collision probability is evaluated by arithmetically averaging the collision probability in the saturated and unsaturated periods, it should also be analyzed under the four different conditions which are classified according to the value of $T$ where $T \in(0, \sigma)$, $T \in\left[\sigma, T_{\text {avg }} \sigma\right), T \in\left[T_{\text {avg }} \sigma, T_{\text {avg }} \sigma+T_{D I F S}+T_{\text {trans }}\right)$ and $T \in\left[T_{\text {avg }} \sigma+T_{D I F S}+T_{\text {trans }},+\infty\right)$.

In the first condition where $T \in(0, \sigma)$ and thus the sampling rate is in the range of $(1.15,1.18) \mathrm{Mbps}$, since both the collision probabilities in the saturated and unsaturated periods have nothing to do with the sampling rate, both of them are constants. The overall collision probability, calculated by these two collision probabilities, is therefore displayed as a straight line with the invariable value 0.22 .

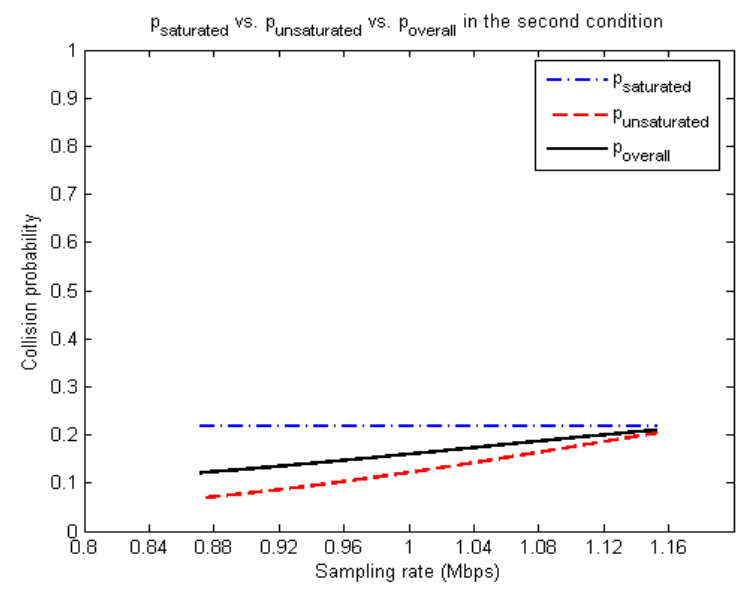

Fig. 5. The collision probability in the second condition where $T \in$ $\left[\sigma, T_{a v g} \sigma\right)$

In the second condition where $T \in\left[\sigma, T_{a v g} \sigma\right)$, the collision probability in the unsaturated period changes continuously where the sampling rate is in the range of $(0.87,1.15) \mathrm{Mbps}$, thus after averaging it with the collision probability in the saturated period, the overall collision probability behaves as a smoother curve, shown in Fig. 5.

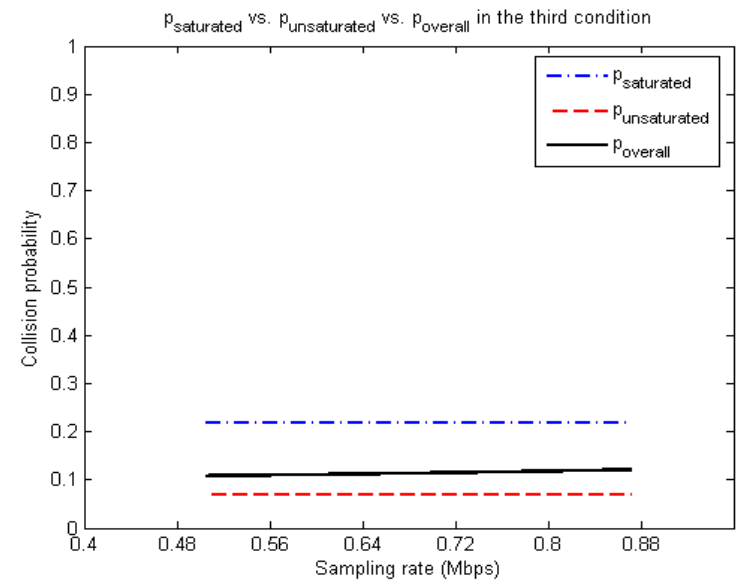

Fig. 6. The collision probability in the third condition where $T \in$ $\left[T_{a v g} \sigma, T_{a v g} \sigma+T_{D I F S}+T_{\text {trans }}\right)$

In the third condition where the sampling rate varies from $0.51 \mathrm{Mbps}$ to $0.87 \mathrm{Mbps}$, the collision probability in both the saturated and unsaturated periods are invariables with the value 0.22 and 0.068 , respectively. Nevertheless, due to the fact that the weight used for the average is sampling rate dependent, the overall collision probability acts as a curve rather than a straight line, as Fig. 6 displays. 


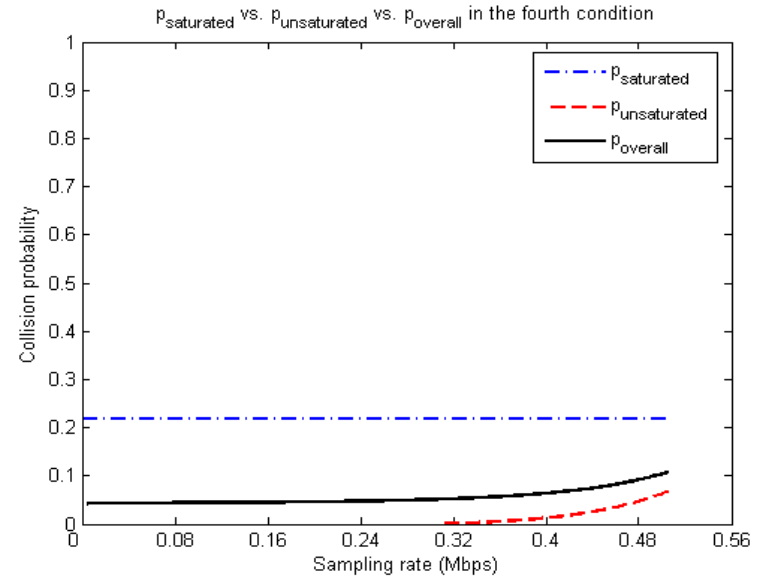

Fig. 7. The collision probability in the fourth condition where $T \in\left[T_{a v g} \sigma+\right.$ $\left.T_{\text {DIFS }}+T_{\text {trans }},+\infty\right)$

In the fourth condition where $T \in\left[T_{a v g} \sigma+T_{D I F S}+\right.$ $\left.T_{\text {trans }},+\infty\right)$ and thus the sampling rate belongs to $(0,0.51)$ Mbps, affected by the sampling rate, the collision probability in the unsaturated period behaves as a curve which begins to emerge when the sampling rate exceeds $0.3 \mathrm{Mbps}$, as displayed in Fig. 7. This indicates that collision will never occur among sensor nodes in the unsaturated period if the sampling rate is smaller than 0.3 Mbps. So after averaging, the overall collision probability appears as a curve and starts from zero Mbps. The reason is that the collision probability in the saturated period remains nonzero in the sampling rate interval $(0,0.3) \mathrm{Mbps}$.

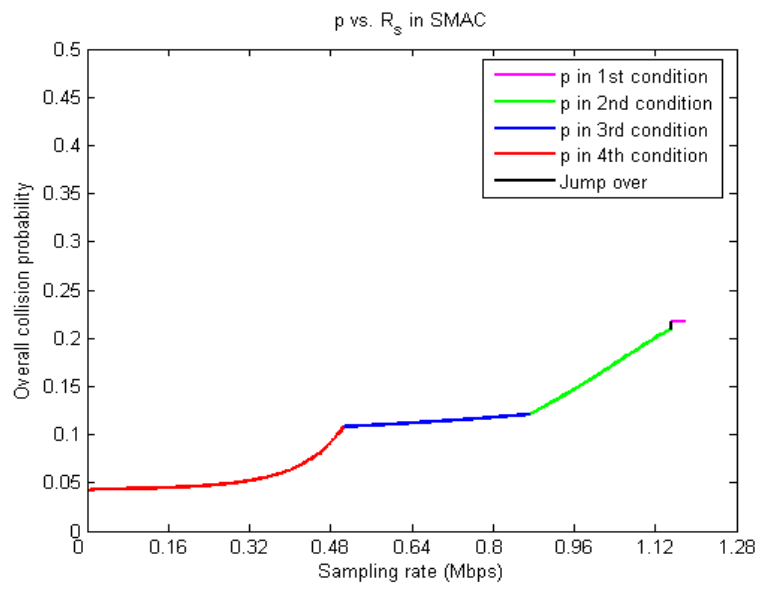

Fig. 8. Overall collision probability in the SMAC protocol.

In order to give an overall look of the performance of the collision probability in the whole listen phase, we combine all these four figures together. Deserving to be mentioned, there is a gap standing between the lines of the average collision probability in the first and second conditions, which is denoted by a small black line. The reason of this phenomenon is that the method of evaluating one of the parameters (active neighbor node) which determine the value of the collision probability in the unsaturated period is different in these two conditions [12]. As a result, the overall collision probability jumps a big step directly from 0.21 to 0.22 once the sampling rate increases to $1.15 \mathrm{Mbps}$, as described in Fig. 8.

\section{CONCLUSION}

In this paper, we evaluate the collision probability of hybrid MAC protocols in wireless sensor networks by considering both the saturated and the unsaturated situations. We assert that the collision probability is highly affected by the sampling rate and varies from 0.043 to 0.22 when the sampling rate changes from zero to $1.18 \mathrm{Mbps}$. Consequently, in terms of the characteristics of the collision probability, the collision avoidance technique can be removed once the sampling rate is relatively low according to the performance requirements of the wireless sensor applications.

\section{REFERENCES}

[1] W. Dargie, X. Chao and M. K. Denko, "Modelling the energy cost of a fully operational wireless sensor network," Telecommunication Systems, Springer Netherlands, 2009.

[2] S. Kim, S. Pakzad, D. Culler, J. Demmel, G. Fenves, S. Glaser and M. Turon, "Health monitoring of civil infrastructures using wireless sensor networks," Proceedings of the 6th international conference on Information processing in sensor networks, pp.254-263. ACM, USA, 2007.

[3] K. Chintalapudi, T. Fu, J. Paek, N. Kothari, S. Rangwala, J. Caffrey, R. Govindan, E. Johnson and S. Masri, "Monitoring civil structures with a wireless sensor network," IEEE Internet Computing, vol.10, pp.26-34, IEEE Educational Activities Department, USA, 2006.

[4] Chao, X., Dargie, W., Lin, G.: Energy model for h2s monitoring wireless sensor network. In: Proceedings of the 2008 11th IEEE International Conference on Computational Science and Engineering, pp:402-409. IEEE Computer Society, USA (2008)

[5] G. Bianchi, "Performance analysis of the IEEE 802.11 distributed coordination function," IEEE Selected Areas in Communications, vol.18, pp.535-547. IEEE Journal on Selected Areas in Communications, 2000.

[6] H. L. Vu and T. Sakurai, "Collision probability in saturated IEEE 802.11 networks," Australian Telecommunication Networks and Applications Conference, Australia, 2006.

[7] L. Ning, "A power-consumption analysis for 802.11DCF," Wireless Communications, Networking and Mobile Computing, pp.1-4. IEEE Conference Proceeding, 2006.

[8] R. Bruno, M. Conti and E. Gregori, "Optimization of efficiency and energy consumption in p-persistent CSMA-based wireless LANs," IEEE Transactions on Mobile Computing, vol.1, pp.10-31. IEEE Educational Activities Department, USA, 2002.

[9] W. Ye, J. Heidemann and D. Estrin, "An energy-efficient MAC protocol for wireless sensor networks," INFOCOM, vol.3, pp.1567-1576. IEEE Proceedings, 2002.

[10] D. Malone, K. Duffy and D. Leith, "Modeling the 802.11 distributed coordination function in nonsaturated heterogeneous conditions," IEEE/ACM Transactions on Networking, vol.15, pp.159-172. IEEE Press, USA, 2007.

[11] A. Kumar, E. Altman, D. Miorandi and M. Goyal, "New insights from a fixed-point analysis of single cell IEEE 802.11 WLANs," IEEE/ACM Transactions on Networking, vol.15, pp.588-601. IEEE Press, USA, 2007.

[12] Q. Dong and W. Dargie, "Analysis of collision probability in unsaturated situation," The 25th ACM Symposium On Applied Computing, pp.774780. Switzerland, 2010.

[13] J. Polastre, J. Hill and D. Culler, "Versatile low power media access for wireless sensor networks," Proceedings of the 2nd international conference on Embedded networked sensor systems, pp.95-107. ACM, USA, 2004.

[14] M. Buettner, G. Yee, E. Anderson and R. Han, "X-MAC: A short preamble MAC protocol for duty-cycled wireless sensor networks," Proceedings of the 4th international conference on Embedded networked sensor systems, pp.307-320. ACM, USA, 2006. 